\title{
Differential evolution algorithm for linear frequency modulation radar signal denoising
}

\begin{abstract}
Signal denoising is directly related to sample estimation of received signals, either by estimating the equation parameters for the target reflections or the surrounding noise and clutter accompanying the data of interest. Radar signals recorded using analogue or digital devices are not immune to noise. Random or white noise with no coherency is mainly produced in the form of random electrons, and caused by heat, environment, and stray circuitry loses. These factors influence the output signal voltage, thus creating detectable noise. Differential Evolution (DE) is an effectual, competent, and robust optimisation method used to solve different problems in the engineering and scientific domains, such as in signal processing. This paper looks at the feasibility of using the differential evolution algorithm to estimate the linear frequency modulation received signal parameters for radar signal denoising. The results gave high target recognition and showed feasibility to denoise received signals.
\end{abstract}

Keyword: Chirp pulse generators; Differential evolution (DE); Target detection; Radar signal denoising 